\title{
3.0 $T$ cardiovascular magnetic resonance in patients treated with coronary stenting for myocardial infarction: evaluation of short term safety and image quality
}

\author{
Robin Nijveldt · Alexander Hirsch • Mark B. M. Hofman · Aernout M. Beek • \\ Anje M. Spijkerboer · Jan J. Piek · Albert C. van Rossum
}

Received: 1 May 2007 / Accepted: 17 August 2007/Published online: 6 September 2007

(C) Springer Science+Business Media B.V. 2007

\begin{abstract}
Purpose To evaluate safety and image quality of cardiovascular magnetic resonance (CMR) at $3.0 \mathrm{~T}$ in patients with coronary stents after myocardial infarction (MI), in comparison to the clinical standard at $1.5 \mathrm{~T}$. Methods Twenty-five patients (21 men; $55 \pm 9$ years) with first MI treated with primary stenting, underwent 18 scans at $3.0 \mathrm{~T}$ and 18 scans at $1.5 \mathrm{~T}$. Twenty-four scans were performed $4 \pm 2$ days and 12 scans $125 \pm 23$ days after MI. Cine (steady-state free precession) and late gadolinium-enhanced (LGE, segmented inversionrecovery gradient echo) images were acquired.
\end{abstract}

R. Nijveldt $(\varangle)$ · A. M. Beek · A. C. van Rossum Department of Cardiology, VU University Medical Center, Room 5F003 De Boelelaan 1117, Amsterdam 1081 HV, The Netherlands

e-mail: R.Nijveldt@vumc.nl

R. Nijveldt · A. Hirsch · A. C. van Rossum Interuniversity Cardiology Institute of the Netherlands, Utrecht, The Netherlands

\section{A. Hirsch · J. J. Piek}

Department of Cardiology, Academic Medical Center, Amsterdam, The Netherlands

M. B. M. Hofman

Department of Physics and Medical Technology, VU University Medical Center, Amsterdam, The Netherlands

\section{A. M. Spijkerboer}

Department of Radiology, Academic Medical Center,

Amsterdam, The Netherlands
Patient safety and image artifacts were evaluated, and in 16 patients stent position was assessed during repeat catheterization. Additionally, image quality was scored from 1 (poor quality) to 4 (excellent quality). Results There were no clinical events within 30 days of CMR at $3.0 \mathrm{~T}$ or $1.5 \mathrm{~T}$, and no stent migration occurred. At $3.0 \mathrm{~T}$, image quality of cine studies was clinically useful in all, but not sufficient for quantitative analysis in $44 \%$ of the scans, due to stent (6/18 scans), flow (7/18 scans) and/or dark band artifacts (8/18 scans). Image quality of LGE images at $3.0 \mathrm{~T}$ was not sufficient for quantitative analysis in $53 \%$, and not clinically useful in $12 \%$. At $1.5 \mathrm{~T}$, all cine and LGE images were quantitatively analyzable. Conclusion $3.0 \mathrm{~T}$ is safe in the acute and chronic phase after MI treated with primary stenting. Although cine imaging at 3.0 $\mathrm{T}$ is suitable for clinical use, quantitative analysis and LGE imaging is less reliable than at $1.5 \mathrm{~T}$. Further optimization of pulse sequences at $3.0 \mathrm{~T}$ is essential.

Keywords Late gadolinium enhancement .

Cardiovascular magnetic resonance.

$3.0 \mathrm{~T} \cdot$ Acute myocardial infarction .

Safety
Abbreviations
CMR Cardiovascular magnetic resonance
LGE Late gadolinium enhancement
SSFP Steady-state free precession
MI Myocardial infarction 


\section{Introduction}

The combination of functional cardiovascular magnetic resonance (CMR) and late gadolinium enhancement (LGE) is evolving as an important diagnostic [1,2] and prognostic [3-5] modality in patients with ischemic heart disease. Because the availability of high field MR systems is increasing, the need arises to evaluate the performance and clinical value of these systems in cardiovascular disease. Earlier reports already suggested that $3.0 \mathrm{~T}$ MR systems offer higher temporal and spatial resolution, due to an increased signal-to-noise ratio, which would be especially advantageous in CMR scanning [6-8]. However, these studies were all assessed in healthy volunteers and in patients with non-ischemic heart disease or suspected coronary artery disease, and without the presence of coronary stents [6-10]. There is limited to no data about safety and image quality at $3.0 \mathrm{~T}$ CMR scanning in the acute or chronic phase after myocardial infarction (MI), in patients treated with percutaneous coronary intervention and primary stenting. In these patients $\mathrm{B}_{0}$ inhomogeneities (induced by the heart-lung interface or from coronary stents), flow artifacts, inhomogeneity of normal myocardial suppression in LGE (induced by $\mathrm{B}_{1}$ inhomogeneity) and poor cardiac triggering may interfere with the gain offered by the higher magnetic field.

The aim of this study was to test whether CMR scanning at 3.0 $\mathrm{T}$ is safe and feasible in patients with coronary stents in the acute and chronic phase after MI, and to prospectively compare image quality at 3.0 $\mathrm{T}$ with the current clinical standard at $1.5 \mathrm{~T}$. Furthermore, the presence and significance of different image artifacts are considered.

\section{Methods}

Patient population

Patients were eligible for the study if they had been admitted with a first ST-elevation acute MI, according to standard electrocardiographic and enzymatic criteria [11], and had undergone successful primary PCI with stent implantation. Exclusion criteria were electrocardiographic evidence of reinfarction, haemodynamic or other clinical instability or (relative) contraindications for CMR such as claustrophobia, pacemakers, intracerebral aneurysm clips or very irregular heart rhythm. Patients were treated with aspirin, heparin, abciximab, clopidogrel, statins, beta-blockade and ACE-inhibitors, according to ACC/AHA practice guidelines [12]. The study was approved by the local ethics committee and all patients gave written informed consent.

\section{CMR parameters}

Eighteen CMR scans were performed with a $3.0 \mathrm{~T}$ MR system (Intera, Philips, Best, The Netherlands), with a gradient performance of $30 \mathrm{mT} / \mathrm{m}$ and slew rate of $150 \mathrm{~T} / \mathrm{m} / \mathrm{s}$, using a six element cardiac phased array surface coil. Another $18 \mathrm{CMR}$ scans were acquired with a 1.5 T MR system (Magnetom Sonata, Siemens, Erlangen, Germany), with a gradient performance of $40 \mathrm{mT} / \mathrm{m}$ and slew rate of $200 \mathrm{~T} / \mathrm{m} / \mathrm{s}$, using an eight element cardiac phased array surface coil.

Cine imaging was performed at both field strengths using a SSFP pulse sequence, without parallel imaging. Long axis views, as well as short axis views covering the entire left ventricle were acquired during repeated breath-holds in expiration. At $3.0 \mathrm{~T}$, cine SSFP sequence parameters were a temporal resolution between $25 \mathrm{~ms}$ and $50 \mathrm{~ms}$, excitation angle of $45^{\circ}$, receiver bandwidth $868 \mathrm{~Hz} /$ pixel, TR/TE of $3.8 / 1.9 \mathrm{~ms}$, matrix $192 \times 155$ and voxel size of $1.5 \times 1.8 \times 6.0 \mathrm{~mm}^{3}$. At $1.5 \mathrm{~T}$, the temporal resolution was between $35 \mathrm{~ms}$ and $50 \mathrm{~ms}$ with an excitation angle of $60^{\circ}$, receiver bandwidth $930 \mathrm{~Hz} /$ pixel, TR/TE of $3.2 / 1.6 \mathrm{~ms}$, matrix $256 \times 156$ and voxel size of $1.4 \times 1.9 \times 6.0 \mathrm{~mm}^{3}$.

LGE images were acquired in mid-diastole using a 2D segmented inversion-recovery gradient-echo pulse sequence, 10-15 min after intravenous injection of $0.2 \mathrm{mmol} / \mathrm{kg}$ of a gadolinium chelate (Dotarem, Guerbet, Roissy, France). The LGE images were obtained in exactly the same orientation as the cine images. Sequence parameters at 3.0 T were an excitation angle of $25^{\circ}$, receiver bandwidth $434 \mathrm{~Hz} /$ pixel, TR/TE of $3.5 / 1.3 \mathrm{~ms}$, matrix $192 \times 119$, voxel size of $1.7 \times 2.1 \times 6.0 \mathrm{~mm}^{3}$, triggering every other heart beat, and an inversion time between $250 \mathrm{~ms}$ and $350 \mathrm{~ms}$ to null remote myocardium. At $1.5 \mathrm{~T}$, sequence parameters were an excitation angle of $25^{\circ}$, receiver bandwidth $130 \mathrm{~Hz} /$ pixel, TR/TE of 
9.6/4.4 ms, matrix $256 \times 166$, voxel size of $1.4 \times 1.7 \times 6.0 \mathrm{~mm}^{3}$, triggering every other heart beat, and an inversion time between $220 \mathrm{~ms}$ and $300 \mathrm{~ms}$ to null remote myocardium.

Safety and quality analysis

To evaluate patient safety, a physician was present at the CMR scanner throughout the scan. Heart rhythm was monitored continuously. The patient was asked to report any discomfort and symptoms during the scan procedure. Additionally, a repeat catheterization was performed in 16 patients who underwent CMR examination in the acute phase after MI ( 9 at $3.0 \mathrm{~T}, 7$ at $1.5 \mathrm{~T})$. Stent position and patency after CMR scanning were visually assessed on the repeat catheterization and compared to the primary PCI. Occurrence of repeat intervention or hospitalization within 30 days of the CMR examination was recorded.

Different types of artifacts were reviewed and marked as being clinically relevant if the artifact interfered with visualisation of the myocardium. Flow related artifacts in SSFP imaging [13], and artifacts due to static field inhomogeneities such as the heart-lung interface were scored on cine images. Separately, we looked at artifacts caused by the coronary stent on the cine images, and measured the maximum artifact diameter, perpendicular to the length of the stent, using an appropriate cardiac view. In addition, the potential effect of $\mathrm{B}_{1}$ inhomogeneity on the homogeneous suppression of viable myocardium was visually assessed on LGE images.

Furthermore, image quality of the cine and LGE images were scored on a separate workstation (Centricity Radiology v6.1, GE Medical Systems, Zeist, the Netherlands) by four independent observers, who were blinded for MR system and clinical history. To distinguish between image quality that is satisfactory for clinical use or high quality images for research purposes, images were scored on a scale from 1 to 4: 1 not clinically useful; 2 clinically useful, but of insufficient quality for quantitative analysis; 3 clinically useful and of sufficient quality for quantitative analysis; 4 excellent quality. The following definitions were used for evaluation of the cine images: 1 poor quality, extensive artifacts, completely obscuring endocardial borders; 2 moderate quality, assessment of global function is possible, partly using assumptions, but regional wall thickening is not possible in all segments, due to interfering artifacts; 3 good quality, assessment of global and regional function is possible, despite some small artifacts; 4 excellent quality, functional analysis is possible and there is no interference of artifacts. And for the evaluation of LGE images: 1 poor quality, extensive artifacts, infarcted myocardium is not visible; 2 moderate quality, infarcted myocardium is visible, but delineation is not possible in all segments; 3 good quality, infarcted myocardium is easy to distinguish from viable myocardium, despite some small artifacts; 4 excellent quality, no artifacts. Two scores were given for each scan: 1 for the set of cine images and 1 for the set of LGE images. After completing the independent image quality assessment, all 4 observers exchanged their scores for each case and agreed on a consensus score.

\section{Statistical analysis}

Continuous variables with normal distribution are expressed as mean $\pm \mathrm{SD}$, or as median (25th-75th percentile). Comparison of the baseline characteristics and stent artifact diameter was done by using an unpaired Student's $t$-test. The consensus score of image quality of both field strengths was assessed for statistical difference by a Mann-Whitney $U$ test. All statistical tests were two-sided with a significance level of $P<0.05$. SPSS 12.0.1 for Windows (SPSS Inc., Chicago, USA) was used for analysis.

\section{Results}

Twenty-five consecutive patients with 36 CMR studies were included in the study. One patient was studied on both MR systems in the acute phase, 9 patients ( 5 on $3.0 \mathrm{~T}, 4$ on $1.5 \mathrm{~T}$ ) were studied both in the acute phase and in the chronic phase, and 1 patient $(3.0 \mathrm{~T})$ was studied twice in the chronic phase after MI. The baseline characteristics of all 25 patients are listed in Table 1 . On each system, 12 CMR scans were performed in the acute phase after $\mathrm{MI}$, at $4 \pm 2$ days after primary PCI, and $6 \mathrm{CMR}$ studies in the chronic phase, at $125 \pm 23$ days after 
primary PCI. One patient refused contrast injection during scanning in the chronic phase.

ECG electrodes were positioned and displaced until an optimal ECG signal was acquired. Although it took more time to obtain a stable ECG waveform with a clearly delineated $\mathrm{R}$ wave at $3.0 \mathrm{~T}$ than at $1.5 \mathrm{~T}$ (10-15 min vs. $<2$ min respectively), it did not affect image quality, since scans were repeated when trigger problems occurred.

Stent safety and artifacts at $3.0 \mathrm{~T}$

No patient reported any discomfort or symptoms during the CMR scan procedure. In addition, there were no clinical events during, or shortly after scanning at 3.0 T, and none of the patients underwent a repeat intervention or hospitalization for any reason within 30 days.

The mean number of stents implanted per patient per scan at $3.0 \mathrm{~T}$ was $1.3 \pm 0.7$, with a mean stent length of $19 \pm 5 \mathrm{~mm}$ and diameter of $3.2 \pm 0.4 \mathrm{~mm}$. Further specifications of the implanted coronary stents are listed in Table 2. Repeat catheterization was performed $2(1-4)$ days after scanning in 9 of the 12 patients who underwent 3.0 T CMR scanning in the acute phase after MI. Angiographic evaluation revealed no differences in stent position and patency compared to the initial result after primary PCI.

Table 1 Patient characteristics

\begin{tabular}{ll}
\hline Number of patients & 25 \\
Age (years) & $55 \pm 9$ \\
Men & $21(84)$ \\
BMI $\left(\mathrm{kg} / \mathrm{m}^{2}\right)$ & $25.5 \pm 1.9$ \\
Risk factors & \\
Diabetes mellitus & $0(0)$ \\
Hyperlipidaemia & $3(12)$ \\
Hypertension & $6(24)$ \\
Smoking & $16(64)$ \\
Family history of CAD & $8(32)$ \\
Maximum peak CK-MB (U/L) & $308(197-464)$ \\
Infarct-related artery & \\
LAD & $18(72)$ \\
RCx & $3(12)$ \\
RCA & $4(16)$
\end{tabular}

Values are presented as number (ratio in \%), mean \pm standard deviation or median (25th-75th percentile)
Table 2 Type and number of coronary stents used per CMR examination

\begin{tabular}{|c|c|c|}
\hline $\begin{array}{l}\text { Field strength } \\
\text { and timing }\end{array}$ & $\begin{array}{l}\text { Number of } \\
\text { patients }\end{array}$ & $\begin{array}{l}\text { Number and } \\
\text { type of coronary } \\
\text { stents present during } \mathrm{CMR}^{\mathrm{a}}\end{array}$ \\
\hline \multirow[t]{3}{*}{$3.0 \mathrm{~T}$ acute phase } & 9 & $1 \times$ Multi-Link Vision \\
\hline & 1 & $2 \times$ Multi-Link Vision \\
\hline & 2 & $1 \times$ Driver \\
\hline \multirow[t]{3}{*}{ 3.0 $\mathrm{T}$ chronic phase } & 3 & $1 \times$ Multi-Link Vision \\
\hline & 2 & $\begin{array}{l}\text { 1× Driver/2x TAXUS } \\
\text { Liberté }^{\mathrm{b}}\end{array}$ \\
\hline & 1 & $1 \times$ Driver \\
\hline \multirow[t]{8}{*}{ 1.5 $\mathrm{T}$ acute phase } & 4 & $1 \times$ Prokinetic \\
\hline & 1 & $2 \times$ Prokinetic \\
\hline & 2 & $1 \times$ Lekton Motion \\
\hline & 1 & $\begin{array}{c}1 \times \text { Lekton Motion/2× } \\
\text { Multi-Link Vision }\end{array}$ \\
\hline & 1 & $1 \times$ Multi-Link Vision \\
\hline & 1 & $1 \times \mathrm{AVE}$ \\
\hline & 1 & 1× Multi-Link Zeta \\
\hline & 1 & $1 \times$ CYPHER $^{b}$ \\
\hline \multirow[t]{5}{*}{ 1.5 $\mathrm{T}$ chronic phase } & 2 & $1 \times$ Lekton Motion \\
\hline & 1 & $2 \times$ Lekton Motion \\
\hline & 1 & $1 \times \mathrm{AVE}$ \\
\hline & 1 & 1× Multi-Link Zeta \\
\hline & 1 & $1 \times$ CYPHER $^{\mathrm{b}}$ \\
\hline
\end{tabular}

${ }^{a}$ Stent material and manufacturer-Multi-Link Vision: cobalt chromium alloy, Abbott Vascular; Driver, cobalt chromium alloy, Medtronic; TAXUS Liberté, 316L stainless steel, Boston Scientific; Prokinetic, cobalt chromium alloy, Biotronik; Lekton Motion: 316L stainless steel, Biotronik; Multi-Link Zeta, 316L stainless steel, Abbott Vascular; AVE: 316L stainless steel, Medtronic; CYPHER: 316L stainless steel, Cordis

b Drug-eluting stent

During cine imaging, the coronary stent was visible in 14 of the 18 scans (78\%), with a susceptibility related signal loss of $12.8 \pm 4.3 \mathrm{~mm}$ in diameter. Due to severe interference of the coronary stent in 6 scans (33\%), analysis of surrounding myocardium was impossible (Fig. 1A). Through-plane flow artifacts were present in 14 scans (from the aorta in 9 scans, from the pulmonary trunk in 7 scans, from the left ventricle in 3 scans), and hindered visualization of the myocardium in 7 scans (39\%). In-plane flow artifacts were present in 4 scans, but were not clinically relevant. In the majority of the scans (94\%) dark band artifacts appeared at the 
Fig. 1 Short axis cine SSFP images in different patients, demonstrating a signal void from a coronary stent with myocardial interference at $3.0 \mathrm{~T}$ (A, white arrow head), and a smaller signal void without interference at $1.5 \mathrm{~T}(\mathbf{B}$, white arrow head $)$
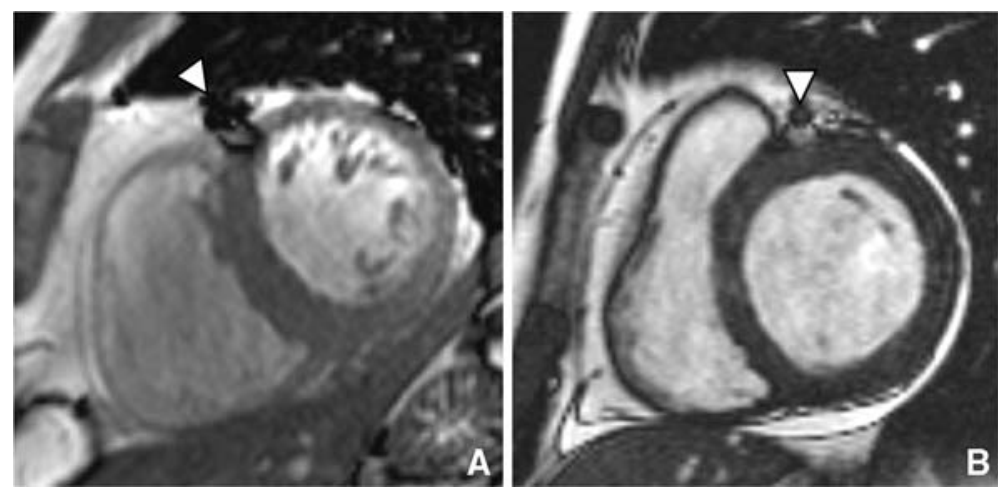

transition between myocardium and lung, with a predilection for the anterolateral wall, which resulted in severe image distortion in 8 scans (44\%). Overall, artifacts were responsible for clinically relevant image deformation in 12 scans (67\%), of which half was caused by the coronary stent (in 6 scans).

No effect could be observed of $B_{1}$ inhomogeneity on the suppression of viable myocardium on LGE images at $3.0 \mathrm{~T}$.

\section{Stent safety and artifacts at $1.5 \mathrm{~T}$}

Also during scanning at $1.5 \mathrm{~T}$, no patient reported any discomfort or symptoms, and there were no clinical events during, or shortly after scanning. None of the patients underwent a repeat intervention or hospitalization for any reason within 30 days after CMR scanning.

The mean number of implanted stents per patient per scan at $1.5 \mathrm{~T}$ was $1.2 \pm 0.5$ (see also Table 2). The mean stent length was $19 \pm 5 \mathrm{~mm}$ with a mean diameter of $3.2 \pm 0.4 \mathrm{~mm}$, which was comparable with the stent length and diameter scanned at $3.0 \mathrm{~T}$ ( $\mathrm{p}=\mathrm{ns}$ ). In 7 of the 12 patients who underwent CMR scanning at $1.5 \mathrm{~T}$ in the acute phase, repeat catheterization at $2(2-4)$ days after scanning revealed no differences in stent position and patency compared to post-PCI.

During cine imaging, the coronary stent was visible in 6 of the 18 scans (33\%), with a susceptibility related signal loss of $5.5 \pm 0.3 \mathrm{~mm}$ in diameter, which was significantly smaller than at $3.0 \mathrm{~T}$ $(P<0.01)$. Furthermore, visible stents did not cause clinically important image deformation (Fig. 1B).
Besides the small signal void from coronary stents, no other artifacts were observed at $1.5 \mathrm{~T}$.

Also at $1.5 \mathrm{~T}$ there was no effect of $\mathrm{B}_{1}$ inhomogeneity on the suppression of viable myocardium on LGE images.

Evaluation of image quality

With scanning at $3.0 \mathrm{~T}$, localized shimming was compulsory in every scan to obtain better image quality (Fig. 2). At $1.5 \mathrm{~T}$, there was no need for adjustments in sequence parameters or the use of local shimming to optimize image quality. At $3.0 \mathrm{~T}$, in $44 \%$ of the cases, image quality was not sufficient for quantitative analysis and assessment of regional function of the left ventricle (Table 3). For clinical purposes and global assessment of left ventricular function, image quality at $3.0 \mathrm{~T}$ and $1.5 \mathrm{~T}$ were comparable. Image quality of LGE images was not sufficient for quantitative analysis in 53\% of the cases at $3.0 \mathrm{~T}$, and $12 \%$ of the images were not useful for clinical purposes. Both image quality of cine and LGE imaging at $3.0 \mathrm{~T}$ were significantly lower compared to $1.5 \mathrm{~T}(P<0.001)$.

\section{Discussion}

In this paper we report on our initial experience with CMR scanning at $3.0 \mathrm{~T}$ in a clinical situation. We found that it is safe and feasible to perform CMR scanning at 3.0 $\mathrm{T}$ in the acute and chronic phase after MI in patients treated with primary stenting. Image quality of cine imaging at $3.0 \mathrm{~T}$ is of sufficient quality for global assessment of left ventricular 
Fig. 2 Four chamber cine SSFP images at $3.0 \mathrm{~T}$ in end-diastole $(\mathbf{A} \& \mathbf{C})$ and end-systole (B \& D), at the same slice position in one patient. Upper panels are without and lower panels with localized shimming. Dark band artifacts (apex) and flow artifacts (around the atrioventricular valves) are reduced with localized shimming
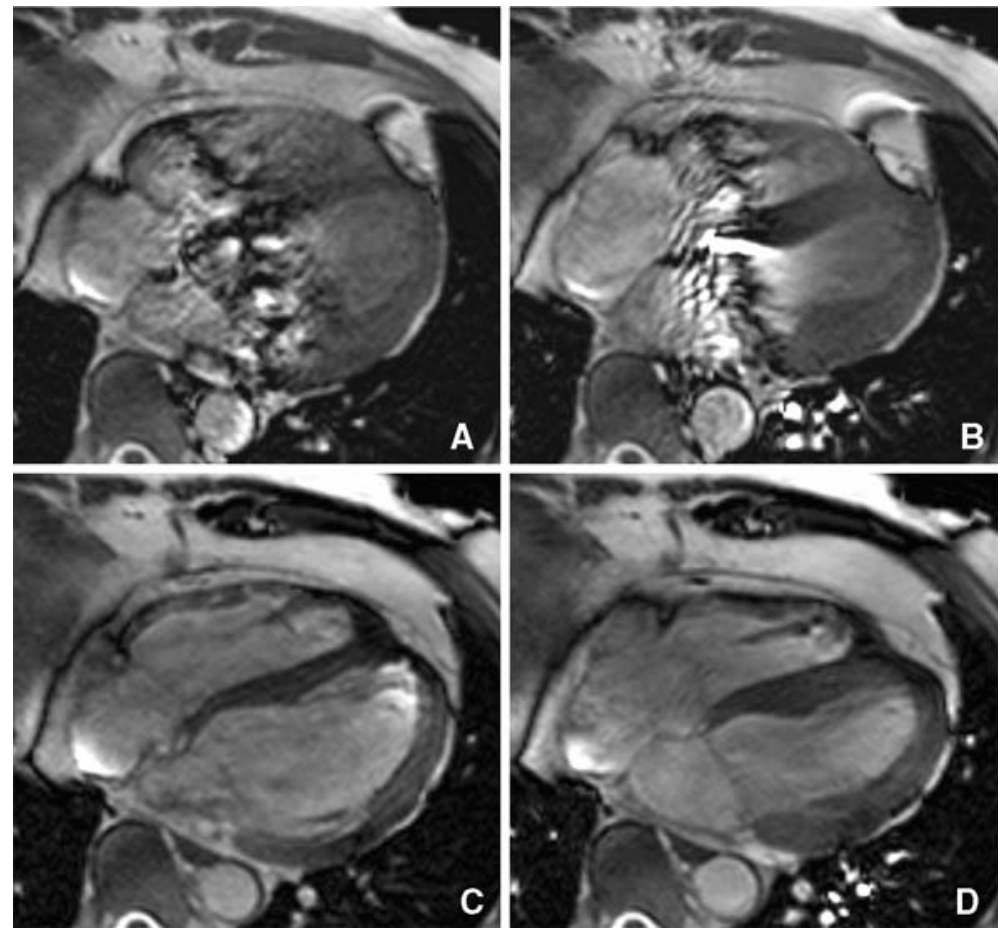

Table 3 Consensus score of image quality of cine and late gadolinium-enhanced images for $1.5 \mathrm{~T}$ and $3.0 \mathrm{~T}$

\begin{tabular}{llllll}
\hline & \multicolumn{2}{l}{ Cine SSFP images } & & \multicolumn{2}{l}{ Late gadolinium-enhanced images } \\
\cline { 2 - 3 } & $1.5 \mathrm{~T}$ & $3.0 \mathrm{~T}$ & & $1.5 \mathrm{~T}$ & $3.0 \mathrm{~T}$ \\
\hline 1. Not clinically useful & $0(0 \%)$ & $0(0 \%)$ & & $0(0 \%)$ & $2(12 \%)$ \\
2. Clinically useful, no quantitative analysis & $0(0 \%)$ & $8(44 \%)$ & & $0(0 \%)$ & $7(41 \%)$ \\
3. Clinically useful, quantitatively analyzable & $0(0 \%)$ & $4(22 \%)$ & & $1(6 \%)$ & $8(47 \%)$ \\
4. Excellent quality & $18(100 \%)$ & $6(33 \%)$ & & $17(94 \%)$ & $0(0 \%)$ \\
\hline
\end{tabular}

Values are presented as absolute numbers (percentage)

function, however, quantitative analysis is not possible in almost half of the patients, due to dark band, flow and stent artifacts. Image quality of LGE studies were significantly better at $1.5 \mathrm{~T}$. Therefore further optimization of pulse sequences at $3.0 \mathrm{~T}$ is essential.

MR imaging is considered to be contraindicated in patients with ferromagnetic implants, primarily because of the potential risks associated with migration, the induction of an electrical current and heating of the implant [14, 15]. According to earlier reports and the American Society for Testing and Materials International, the overall magnetic field interaction and heating for coronary stents is limited or absent at $1.5 \mathrm{~T}$ and $3.0 \mathrm{~T}$, as tested in vitro [16-18]. In vivo studies concerning stent safety at $1.5 \mathrm{~T}$ demonstrated that CMR scanning is safe in patients early after coronary artery stent placement [19-21]. In this in vivo study using both $1.5 \mathrm{~T}$ and $3.0 \mathrm{~T}$, no clinical signs were observed by the attending physician, and no substantial side effects or clinical events occurred during or within 30 days of CMR scanning. In addition, in the patients who underwent a repeat catheterization there was no angiographic evidence of stent migration, confirming the in vitro data.

As discussed by Schär et al. [7], dark band and flow artifacts in SSFP cine imaging can be solved using optimized sequence parameters, localized shimming and correct water resonance frequency adjustment. In the clinical setting of the present study, it was not always possible to solve these 
Fig. 3 Short axis cine SSFP image (A) and cine spoiled gradient-echo image (B), at the same slice position in one patient at 3.0 T. The coronary stent artifact on a SSFP image is larger than on a spoiled gradient-echo image (white arrows heads). Flow artifacts (A, asterisk) are less visible with spoiled gradient-echo imaging at $3.0 \mathrm{~T}$
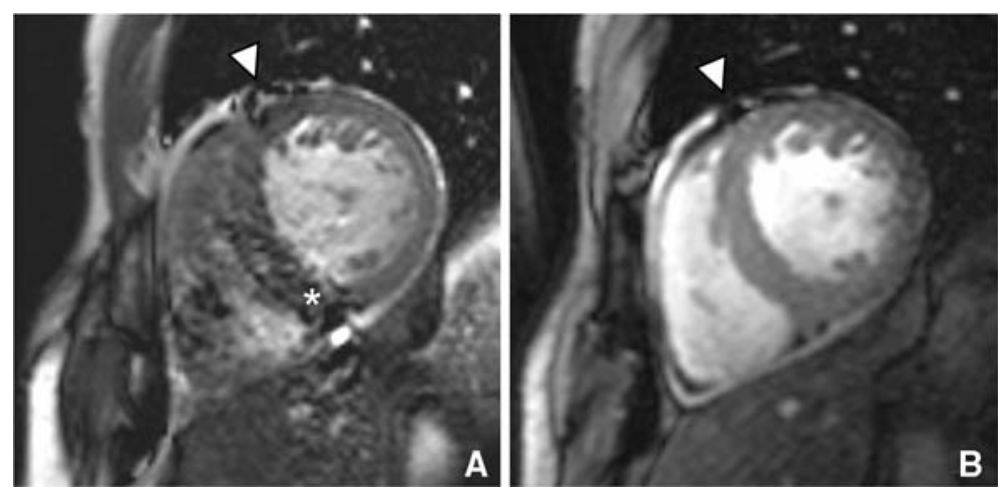

artifact problems despite sequence optimization and localized shimming, especially in the presence of stent artifacts. The larger and diagnostically interfering artifacts of coronary stents at $3.0 \mathrm{~T}$ as opposed to the smaller stent artifacts at $1.5 \mathrm{~T}$ are an important issue in patients after MI, since primary PCI with stent implantation is the method of choice to reestablish coronary flow [22]. For clinical application, it may be more advantageous to return to spoiled gradient-echo cine imaging at $3.0 \mathrm{~T}$, which includes longer acquisition times and lower contrast between blood and myocardium, but is less sensitive for offresonance artifacts and equally accurate as SSFP cine imaging (Fig. 3) [9, 23].

We initially intended to evaluate differences in signal- and contrast-to-noise ratios (SNR and CNR) of both pulse sequences as well. At $1.5 \mathrm{~T}$ good noise estimates could be made using the technique described by Constantinides et al. [24]. However, this method did not work at $3.0 \mathrm{~T}$ due to a different reconstruction algorithm, even without parallel imaging, and by switching off clear and image enhancement filters. Subtraction methods with two consecutive scans to estimate noise are inaccurate in a mobile tissue as the heart [25]. Other methods were beyond the scope of this initial study. Despite the fact that SNR's and CNR's were therefore omitted from this study, it is interesting to mention that an important reason for inferior quality of LGE images at 3.0 T was that infarcted myocardium was sometimes difficult to delineate from the left ventricular cavity (Fig. 4).

We used the same contrast agent and dose at both field strengths. The contrast dose has been optimized for $1.5 \mathrm{~T}$ in the past [26, 27], but might be different at $3.0 \mathrm{~T}$ as $\mathrm{T} 1$ relaxation rates are in general field strength dependent. However, a recent study of Sharma and colleagues showed that there were only minor differences in post contrast myocardial $\mathrm{T} 1$ relaxation times between $1.5 \mathrm{~T}$ and $3.0 \mathrm{~T}$, using a contrast dose of $0.2 \mathrm{mmol} / \mathrm{kg}$ [28]. As long as inversion times are set appropriately at each field strength as done in this study, it is not to be expected that significant differences in LGE image quality are caused by contrast dose effects.

In conclusion, our study demonstrates that it is safe and feasible to perform CMR scanning at $3.0 \mathrm{~T}$ in the
Fig. 4 Short axis LGE images $15 \mathrm{~min}$ after injection of contrast at $3.0 \mathrm{~T}(\mathbf{A})$ and $1.5 \mathrm{~T}(\mathbf{B})$, in different patients. Visual assessment of infarct extent and location was more difficult at $3.0 \mathrm{~T}$ than at $1.5 \mathrm{~T}$, since it was sometimes difficult to delineate infarcted myocardium from the left ventricular cavity at $3.0 \mathrm{~T}$
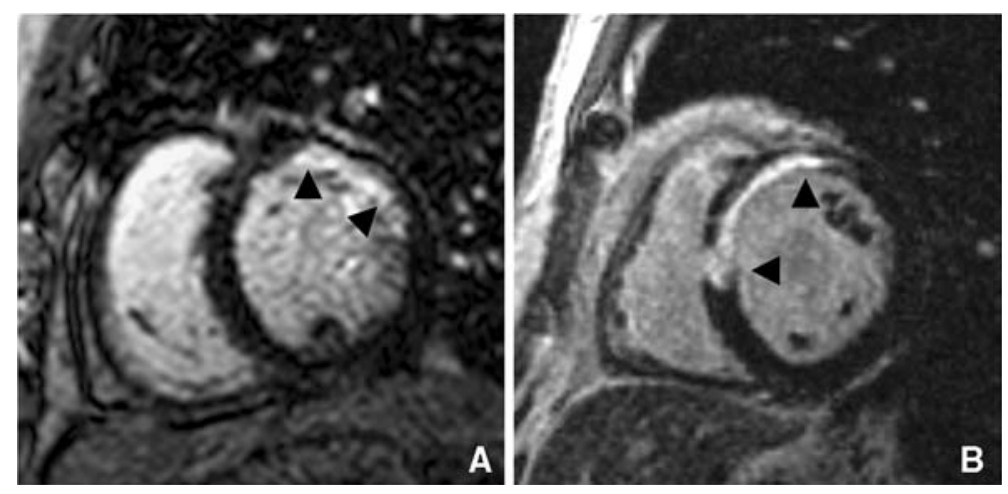
acute and chronic phase after MI in patients treated with primary stenting. Although cine imaging at $3.0 \mathrm{~T}$ is of sufficient quality for clinical use, quantitative assessment is less reliable compared to $1.5 \mathrm{~T}$, mainly due to dark band, flow and stent artifacts. Further optimization of pulse sequences at $3.0 \mathrm{~T}$ is essential to make $3.0 \mathrm{~T}$ CMR scanning suitable for clinical cardiology.

\section{Limitations}

The use of different MR systems, from different vendors, with different coils and sequence parameters, of course introduces confounding factors for a comparison. However, the sequences were optimized for their field strength to evaluate safety, feasibility and image quality rather than technical differences. A second limitation is that patients did not undergo a CMR examination at both $1.5 \mathrm{~T}$ and $3.0 \mathrm{~T}$. Because the MR systems were on two different locations, it was not feasible to study the same patient twice in the acute phase after MI.

Acknowledgements This study was supported by Netherlands Heart Foundation grant 2003B126 from the Netherlands Heart Foundation. We thank Guerbet Nederland B.V. (Gorinchem, the Netherlands) for providing the contrast agent used in this study.

\section{References}

1. Pennell DJ, Sechtem UP, Higgins CB et al (2004) Clinical indications for cardiovascular magnetic resonance (CMR): consensus panel report. Eur Heart J 25(21):1940-1965

2. Schietinger BJ, Voros S, Isbell DC et al (2007) Can late gadolinium enhancement by cardiovascular magnetic resonance identify coronary artery disease as the etiology of new onset congestive heart failure? Int $\mathrm{J}$ Cardiovasc Imaging, doi: 10.1007/s10554-006-9200-X

3. Wu KC, Zerhouni EA, Judd RM et al (1998) Prognostic significance of microvascular obstruction by magnetic resonance imaging in patients with acute myocardial infarction. Circulation 97(8):765-772

4. Kim RJ, Wu E, Rafael A et al (2000) The use of contrastenhanced magnetic resonance imaging to identify reversible myocardial dysfunction. N Engl J Med 343(20):14451453

5. Beek AM, Kuhl HP, Bondarenko O et al (2003) Delayed contrast-enhanced magnetic resonance imaging for the prediction of regional functional improvement after acute myocardial infarction. J Am Coll Cardiol 42(5):895-901

6. Hinton DP, Wald LL, Pitts J et al (2003) Comparison of cardiac MRI on 1.5 and 3.0 Tesla clinical whole body systems. Invest Radiol 38(7):436-442
7. Schar M, Kozerke S, Fischer SE et al (2004) Cardiac SSFP imaging at 3 Tesla. Magn Reson Med 51(4):799-806

8. Gutberlet M, Schwinge K, Freyhardt P et al (2005) Influence of high magnetic field strengths and parallel acquisition strategies on image quality in cardiac $2 \mathrm{D}$ CINE magnetic resonance imaging: comparison of $1.5 \mathrm{~T}$ vs. 3.0 T. Eur Radiol 15(8):1586-1597

9. Michaely HJ, Nael K, Schoenberg SO et al (2006) Analysis of cardiac function-comparison between 1.5 Tesla and 3.0 Tesla cardiac cine magnetic resonance imaging: preliminary experience. Invest Radiol 41(2):133-140

10. Gutberlet M, Noeske R, Schwinge K et al (2006) Comprehensive cardiac magnetic resonance imaging at 3.0 Tesla: feasibility and implications for clinical applications. Invest Radiol 41(2):154-167

11. Alpert JS, Thygesen K, Antman E et al (2000) Myocardial infarction redefined - a consensus document of The Joint European Society of Cardiology/American College of Cardiology Committee for the redefinition of myocardial infarction. J Am Coll Cardiol 36(3):959-969

12. Ryan TJ, Antman EM, Brooks NH et al (1999) 1999 update: ACC/AHA guidelines for the management of patients with acute myocardial infarction: executive summary and recommendations: a report of the American College of Cardiology/American Heart Association Task Force on Practice Guidelines (Committee on Management of Acute Myocardial Infarction). Circulation 100(9):1016-1030

13. Markl M, Pelc NJ (2004) On flow effects in balanced steady-state free precession imaging: pictorial description, parameter dependence, and clinical implications. J Magn Reson Imaging 20(4):697-705

14. New PF, Rosen BR, Brady TJ et al (1983) Potential hazards and artifacts of ferromagnetic and nonferromagnetic surgical and dental materials and devices in nuclear magnetic resonance imaging. Radiology 147(1):139-148

15. Shellock FG, Crues JV (1988) High-field-strength MR imaging and metallic biomedical implants: an ex vivo evaluation of deflection forces. Am $\mathrm{J}$ Roentgenol 151(2):389-392

16. ASTM International: F2182 (2003) Test method for measurement of radio frequency induced heating near passive implants during magnetic resonance imaging. American Society for Testing and Materials (ASTM) International, West Conshohocken, PA

17. Hug J, Nagel E, Bornstedt A et al (2000) Coronary arterial stents: safety and artifacts during MR imaging. Radiology 216(3):781-787

18. Shellock FG, Forder JR (2005) Drug eluting coronary stent: in vitro evaluation of magnet resonance safety at 3 Tesla. J Cardiovasc Magn Reson 7(2):415-419

19. Gerber TC, Fasseas P, Lennon RJ et al (2003) Clinical safety of magnetic resonance imaging early after coronary artery stent placement. J Am Coll Cardiol 42(7):12951298

20. Schroeder AP, Houlind K, Pedersen EM et al (2000) Magnetic resonance imaging seems safe in patients with intracoronary stents. J Cardiovasc Magn Reson 2(1):43-49

21. Syed MA, Carlson K, Murphy M et al (2006) Long-term safety of cardiac magnetic resonance imaging performed in the first few days after bare-metal stent implantation. J Magn Reson Imaging 24(5):1056-1061 
22. Keeley EC, Boura JA, Grines CL (2003) Primary angioplasty versus intravenous thrombolytic therapy for acute myocardial infarction: a quantitative review of 23 randomised trials. Lancet 361(9351):13-20

23. Barkhausen J, Ruehm SG, Goyen M et al (2001) MR evaluation of ventricular function: true fast imaging with steady-state precession versus fast low-angle shot cine MR imaging: feasibility study. Radiology 219(1):264-269

24. Constantinides CD, Atalar E, McVeigh ER (1997) Signalto-noise measurements in magnitude images from NMR phased arrays. Magn Reson Med 38(5):852-857

25. Price RR, Axel L, Morgan T et al (1990) Quality assurance methods and phantoms for magnetic resonance imaging: report of AAPM nuclear magnetic resonance Task Group No. 1. Med Phys 17(2):287-295
26. Petersen SE, Mohrs OK, Horstick G et al (2004) Influence of contrast agent dose and image acquisition timing on the quantitative determination of nonviable myocardial tissue using delayed contrast-enhanced magnetic resonance imaging. J Cardiovasc Magn Reson 6(2):541-548

27. Wagner A, Mahrholdt H, Thomson L et al (2006) Effects of time, dose, and inversion time for acute myocardial infarct size measurements based on magnetic resonance imaging-delayed contrast enhancement. J Am Coll Cardiol 47(10):2027-2033

28. Sharma P, Socolow J, Patel S et al (2006) Effect of GdDTPA-BMA on blood and myocardial T1 at 1.5T and 3T in humans. J Magn Reson Imaging 23(3):323-330 